\title{
Computational Modelling of Pulsating Biological Flow
}

\author{
X.S. Yang, R.W. Lewis, and H. Zhang \\ Civil and Computational Engineering Centre, School of Engineering, \\ University of Wales Swansea, Swansea SA2 8PP, UK x.s.yang@swansea.ac.uk
}

\begin{abstract}
Computational modelling of biological flow can have many in medicine and biomechanical engineering. Stenosis of blood vessels is the main cause of vascular disease. A mathematical model of pulsating flow through stenotic blood vessels has been formulated. The numerical simulations using finite element analysis show that the axial velocity profile is controlled by the shape of the stenotic plagues and the pulsating pressure gradient. Instability due to the vessel narrowing is closely related non-uniform stress distribution. In addition, the peak value of the shear stress may directly affect the degree and location of the rupture.
\end{abstract}

Keywords: Biological flow, finite element analysis, stenosis.

\section{Introduction}

Vascular disease is mainly caused by the stenosis of fatty plaque deposition and vessel narrowing. As the plaque slowly enlarges, it will cause a significant pressure gradient across the affected region, which may lead to the rupture of the blood vessels. The common clinical observations suggest that the atheromatous disease arise mainly at the bifurcation of arteries. The benefit of modelling this phenomena is obviously profound both in clinical treatment and biomedical research $[1,2,5,6]$. However, most models describing these phenomena only produce some basic features which are far from reality.

This paper aims at using the finite element method to simulate the flow velocity profiles for different shapes of stenotic plague and the possible rupture and patterns under the conditions of pulsating blood flow. The simulation of axial velocity distribution for regular arterial structure show that the rupture is controlled by the shape of the stenotic plaque, the pressure gradient and the amplitude of the pulsating flow. This can form the basis for further research. The implication of the simulation results will also be discussed.

\section{Governing Equations}

The Navier-Stokes equations for incompressible flow can be written as $[3,6]$ :

$$
\nabla \cdot \mathbf{u}=0, \quad \rho\left[\frac{\partial \mathbf{u}}{\partial t}+(\mathbf{u} \cdot \nabla) \mathbf{u}\right]=\mu \nabla^{2} \mathbf{u}-\nabla p
$$


where $\mu$ and $\rho$ are the viscosity and density of the fluid. $\mathbf{u}=(U, V, W)$ and $p$ are flow velocity and pressure of the fluid, respectively. As the blood flow is slow and viscous, so the equations for viscous flow become

$$
\nabla \cdot \mathbf{u}=0, \quad \rho \frac{\partial \mathbf{u}}{\partial t}=\mu \nabla^{2} \mathbf{u}-\nabla p
$$

In terms of the cylindrical coordinates $(r, \theta, z)$, the axial velocity $W(r, z, t)$ and the radial velocity $U(r, z, t)$, the nonlinear terms are negligible $\left(W \frac{\partial W}{\partial z} \ll 1\right)$ in the case of the slow flow in a tube where the axial flow is dominant (i.e., $U \ll$ $W)$. The governing equation for the axial flow can be written in the cylindrical coordinates as

$$
\rho \frac{\partial W}{\partial t}=\mu \frac{1}{r} \frac{\partial}{\partial r}\left(r \frac{\partial W}{\partial r}\right)-\frac{\partial p}{\partial z} .
$$

This problem involves a moving or free boundary at $r=R(z, t)$. To simplify and convert the free boundary problem into a fixed-domain problem, we define a reduced radial coordinate $\xi=r / R(z, t)$, now equation (3) becomes

$$
\rho \frac{\partial W}{\partial t}=\mu\left[\frac{1}{R^{2}} \frac{\partial^{2} W}{\partial \xi^{2}}+\frac{1}{\xi R(z, t)^{2}} \frac{\partial W}{\partial \xi}\right]-\frac{\partial p}{\partial z}+\frac{\rho \xi}{R(z, t)} \frac{\partial R}{\partial t} \frac{\partial W}{\partial \xi} .
$$

The boundary conditions then become

$$
\frac{\partial W}{\partial \xi}=0, \quad \text { at } \xi=0, \quad \mathrm{~W}(\xi, \mathrm{z}, \mathrm{t})=0, \quad \text { at } \xi=1 .
$$

It is clearly seen that this problem has been simplified as the one-dimensional flow problem if the pressure gradient is prescribed. Most earlier studies $[1,2,6]$ assume the following form $\frac{\partial p}{\partial z}=A \cos (\omega t)+\gamma, \quad \frac{\partial p}{\partial r}=0$, where $\gamma$ is the background pressure gradient. In fact, we can assume any known function form for the pressure gradient in terms of a Fourier expansion.

\section{Finite Element Formulation}

From the implementation point of view, the continuity equation can be approximated by a penalty function as $\nabla \cdot \mathbf{u}=-\lambda^{-1} p$ or $p=-\lambda \nabla \cdot \mathbf{u}$ where $\lambda \rightarrow \infty$. For numerical purpose, $\lambda \gg 1$ is a large number $[3,6]$. We now have a general form

$$
\mathbf{M} \dot{\mathbf{u}}=\lambda \mathbf{G u}+\mathbf{K u}+\mathbf{g},
$$

where $\mathbf{M}=\int_{\Omega} \rho N_{i} N_{j} d \Omega$ is the mass matrix, $\mathbf{K}=\int_{\Omega} \nabla N_{i} \mu \nabla N_{j} d \Omega$ is the stiffness matrix, $\mathbf{G}$ is the penalty term, and $\mathbf{g}$ is the term due to body force and natural boundary conditions. If $\lambda \rightarrow \infty$, the requirement $\mathbf{G u} \rightarrow 0$ leads to the trivial solution $\mathbf{u}=0$ if $\mathbf{G}$ is nonsingular. To obtain the nontrivial solution, this implies that $\mathbf{G}$ must be singular. The singularity of $\mathbf{G}$ can be fulfilled by the reduced integration for the $\lambda$-term with a Gauss-Legendre rule of one-order lower than normal rules $[3,4,7]$. 
The nonlinear finite element equation can be solved using the time-stepping and iteration technique. Once the flow velocity $\mathbf{u}$ has been computed at a time level, the pressure $p=-\lambda \nabla \cdot \mathbf{u}$ can be obtained using the smoothing technique for the flux calculation.

\section{Simulations and Results}

By using the finite element method in the previous section, the flow profiles are simulated for a given pulse. We will first focus on the axial velocity distribution, then calculate the mean shear stress at vessel walls and blood flux variation.

\subsection{Axial Velocity Distribution}

The velocity profiles for different stenotic shapes at the peak systole are shown in Figure 1. The left figure is the flow distribution through a tube where there is essentially no stenosis, and thus the velocity is in fact paraboloid. The middle profile is irregular stenosis leading to the distorted velocity profile. The velocity profile on the right is the regular stenosis on the hexagonal shape, and its velocity distribution is correspondingly modulated by the stenosis.
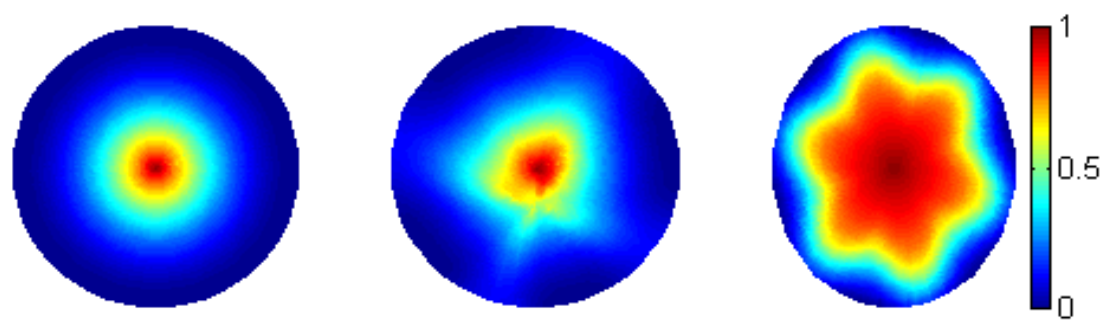

Fig. 1. Velocity profile for different stenotic shapes at the peak systole. The flow velocities are normalized so that $W=0$ at $\xi=1$ and $W=1$ at $\xi=0$.

From these velocity profiles, we can see that the velocity profile is controlled by the shape of the stenosis and thus the blood flux is subsequently affect the degree of the stenosis.

\subsection{Shear Stress and Rupture Prediction}

For a given pulse, one can calculate the wall shear stress and flux variation with time after the peak R-wave of the given pulse. The mean shear stress variation and blood flux variation with time. The characteristics of the computed the shear stress variation is consistent with the experimental results by Oyre et al [6]. It is worth pointing out that there exists a peak for shear stress for a given 
pulsating pressure gradient, and thus the peak shear stress could directly affect the rupture of the stenotic plagues.

Mechanism of rupture of the stenotic plagues is complicated. Generally speaking, the stenosis weakens the lumen of the blood vessel, and thus the rupture could occur if the local shear stress reach to certain value or the yield stress. For a given level of yield stress, the level of shear stress is directly related to the possibility of the rupture. Since the wall shear stress varies with time after the peak R-wave, the peak stress determine the rupture, thus may explain why sudden increase of pulsating pressure could lead to the rupture of weak blood vessels especially near the branch bifurcations.

\section{Discussion}

A mathematical model of pulsating flow through stenotic blood vessels has been formulated, and the finite element method has been implemented after transforming the free boundary problem into a fixed domain problem. Numerical simulations show that the axial velocity profile is controlled by the shapes of the stenotic plagues and pulsating pressure gradient. For a fixed pressure gradient, the velocity profile is distorted by the stenosis.

For a pulsating pressure gradient, the wall shear stress varies with time. It first increases sharply to a peak short after the peak pressure is reached, then it decreases nearly exponentially to a background value. The blood flux also shows the similar characteristics. Thus, the peak value of the shear stress may directly affect the possibility and location of the rupture.

\section{References}

1. Berbich L, Bensalah A, Flaud P and Benkirane, R., Nonlinear analysis of the arterial pulsatile flow: assessment of a model allowing a noninvasive ultrasonic functional exploration, Medical Engineering, 23, 175-183 (2001).

2. Chakravarty, S and Mandal P K, Mathematical modelling of blood flow through an overlapping arterial stenosis, Math. Comput. Modelling,19, 59-70 (1994).

3. Langtangen, H P, Computational Partial Differential Equations: Numerical Methods and Diffpack Programming, Springer, 1999.

4. Lewis R W and Schrefler B A,The finite element method in the static and dynamics deformation and consolidation of porous media, 2nd Ed., John Wiley \& Son, England (1998).

5. Moore J A, Steinman D A, Ethier C R, Computational blood flow modelling: Errors associated with reconstructing finite element models from magnetic resonance images, J. Biomechanics, 31, 179-184 (1998).

6. Oyre S, Ringgaard S, Kozerke, S, Paaske, W, Erlandsen, M, Boesiger, P, and Pedersen, E., Accurate noninvasive quantitation of blood flow, cross-sectional lumen vessel area and wall shear stress by three-dimensional paraboloid modelling of magnetic resonance imaging velocity dat, JACC, 32, 128-134 (1998).

7. Zienkiewicz O C and Taylor R L, The Finite Element Method, vol. I/II, McGrawHill, 4th Edition, 1991. 